\title{
The ligand environment of zinc stored in vesicles
}

\author{
Gerd Wellenreuther ${ }^{a}$, Michele Cianci ${ }^{a}$, Remi Tucoulou ${ }^{b}$, Wolfram Meyer-Klaucke ${ }^{\mathrm{a}, *}$, Hajo Haase ${ }^{\mathrm{c}, *}$ \\ a European Molecular Biology Laboratory, Hamburg Outstation, c/o DESY, Notkestraße 85, D-22603 Hamburg, Germany \\ ${ }^{\mathrm{b}}$ European Synchrotron Radiation Facility, 6 rue Jules Horowitz, Grenoble, France \\ ${ }^{\mathrm{c}}$ Institute of Immunology, Medical Faculty, RWTH Aachen University, Pauwelsstraße 30, D-52074 Aachen, Germany
}

\section{A R T I C L E I N F O}

\section{Article history:}

Received 9 January 2009

Available online $\mathrm{xxxx}$

\section{Keywords:}

Zinc

Metal homeostasis

X-ray absorption spectroscopy

$\mathrm{X}$-ray fluorescence analysis

$\mu$ XANES

Zincosomes

\begin{abstract}
A B S T R A C T
Zinc serves regulatory functions in cells and thus, several mechanisms exist for tight control of its homeostasis. One mechanism is storage in and retrieval from vesicles, so-called zincosomes, but the chemical speciation of zincosomal zinc has remained enigmatic. Here, we determine the intravesicular zinc-coordination in isolated zincosomes in comparison to intact RAW264.7 murine macrophage cells. In elemental maps of a cell monolayer, generated by microbeam X-ray fluorescence, zincosomes were identified as spots of high zinc accumulation. A fingerprint for the binding motif obtained by $\mu$ XANES (X-ray absorption near edge structure) matches the XANES from isolated vesicles; zinc is not free, but present as a complexed form, resembling regulatory or catalytic zinc sites (average coordination: 1.0 sulfur, 2.5 histidines and 1.0 oxygen) in proteins. Such coordination enables reversible binding, acting as a 'zinc sinc', facilitating the accumulation of high amounts of zinc against a concentration gradient.
\end{abstract}

(c) 2009 Elsevier Inc. All rights reserved.
Zinc ions are essential catalytical, structural and co-catalytical components in numerous proteins [1]. Most of these proteins are supplied with zinc in the golgi during synthesis, and frequently the ions stay bound to the protein due to the high affinity of these binding sites. Another group of zinc-binding motifs are regulatory sites, binding zinc reversibly. Thereby, zinc controls the activity of proteins like Metal-responsive element binding transcription factor (MTF)-1 [2] and zinc-uptake regulators [3]. Zinc homeostasis is particularly important for immune function [4] and the concentration of free zinc may act as a signal in immune cells [5].

These regulatory and signaling functions require a tight homeostasis of cytoplasmic free zinc. The total cellular zinc concentration is in the order of several hundred micromolar; most of it proteinbound, leading to low nanomolar or even sub-nanomolar concentrations of free zinc in the cytoplasm of eukaryotic cells. In bacteria even lower, femtomolar concentrations have been described [6]. An intricate system of transport proteins encoded by the SLC30 and SLC39 gene families controls uptake and export of zinc between the cytoplasm and the extracellular space or cellular compartments $[7,8]$. On the other hand, the small cysteine-rich metallothionein binds up to seven zinc ions with different affinities in the nano- to picomolar range, acting as a zinc buffer $[9,10]$. The number of these binding sites is regulated by reversible oxidation

\footnotetext{
* Corresponding authors. Fax: +49 4089902149 (W. Meyer-Klaucke), +49 2418082613 (H. Haase).

E-mail addresses: wolfram@embl-hamburg.de (W. Meyer-Klaucke), hhaase@ ukaachen.de (H. Haase).
}

of metallothioneins thiol residues, connecting redox-metabolism and zinc availability [11].

Another so far only poorly understood regulatory mechanism of the intracellular zinc concentration is its storage in vesicles, socalled 'zincosomes', which have been visualized by staining with fluorescent zinc probes [12]. These vesicles accumulate millimolar amounts of zinc during zinc excess [13] and function as a reversible zinc storage compartment during starvation by serum withdrawal [14]. The main advantage of this kind of storage is the rapid, reversible sequestration of excess zinc, much faster than in case of de novo metallothionein synthesis. While proteins transporting zinc into these vesicles are well known [7], no data exist regarding the chemical speciation of zinc therein. Zincosomal zinc is generally regarded as free or only loosely bound, because it is readily detectable by fluorescent probes like Zinquin and TSQ. However, these probes provide bidentate ligands that can interact with protein-bound zinc, even in metallothionein [15], and their fluorescence is not an indication of zinc presence in a free form. The concentration gradient between zincosomal and cytosolic zinc is a strong driving force for moving zinc out of the vesicles, and thus keeping the metal ions in place should require either a continuous zinc transport with consumption of ATP, or chelation in an intracellular store.

To elucidate the chemical speciation of zincosomal zinc, we followed a 2-fold strategy: (I) Characterization of isolated zinc-accumulating vesicles by X-ray absorption spectroscopy (XAS) and (II) spatial-resolved analysis by micro X-ray fluorescence analysis ( $\mu \mathrm{XRF})$ on frozen, intact cells. Based on the results obtained by both methods we identified a ligand environment in which zinc 
is bound to 1.0 sulfur at a distance of $2.278 \AA$, 2.5 histidines and 1.0 oxygens at $1.968 \AA$. This differs significantly from the binding motifs in other cellular compartments, and further shows that zincosomal zinc does not exist in a free form, but bound to a ligand environment different from water.

\section{Materials and methods}

Cell culture. RAW264.7 murine macrophages were cultured in RPMI1640 medium containing $10 \%$ heat inactivated fetal calf serum, $2 \mathrm{mM} \mathrm{L-glutamine,} 100 \mathrm{U} / \mathrm{ml}$ penicillin, and $100 \mu \mathrm{g} / \mathrm{ml}$ streptomycin. Cells were maintained at $37{ }^{\circ} \mathrm{C}, 100 \%$ humidity, and $5 \% \mathrm{CO}_{2}$.

Fluorescence microscopy. Cells were seeded onto sterile glass coverslips and kept under normal culture conditions or in the presence of $100 \mu \mathrm{M} \mathrm{ZnSO}$ for $24 \mathrm{~h}$. Incubation with the fluorescent zinc indicator TSQ (toluolsulfonamidoquinoline) (Teflabs, Austin, USA) was performed in loading buffer ( $25 \mathrm{mM}$ Hepes, $\mathrm{pH} 7.35$, $120 \mathrm{mM} \mathrm{NaCl}, 5.4 \mathrm{mM} \mathrm{KCl}, 5 \mathrm{mM}$ glucose, $1.3 \mathrm{mM} \mathrm{CaCl}_{2}, 1 \mathrm{mM}$ $\mathrm{MgCl}_{2}, 1 \mathrm{mM} \mathrm{NaH} \mathrm{PO}_{4}, 0.3 \%$ bovine serum albumin) for $30 \mathrm{~min}$ at $37^{\circ} \mathrm{C}$. Fluorescence was monitored with a Zeiss Axioskop and images taken at $40 \times$ magnification using a Nikon Coolpix 4500 digital camera.

Subcellular fractionation. Cells were grown in the presence of $100 \mu \mathrm{M} \mathrm{ZnSO}_{4}$. All isolated subcellular fractions were immediately frozen and kept at cryogenic temperatures.

Vesicles. Cells were lysed by nitrogen cavitation (Nitrogen cell disruption vessel, Parr Instruments, Frankfurt, Germany) as described [16]. The lysate was subjected to centrifugation for $15 \mathrm{~min}$ at $4{ }^{\circ} \mathrm{C}$ and $400 \mathrm{~g}$ to remove nuclei and larger membrane fragments. Organelles were further separated by centrifugation on a discontinuous two step percoll gradient with densities of 1.065 and 1.2 at $37.000 \mathrm{~g}$ for $30 \mathrm{~min}$ at $4{ }^{\circ} \mathrm{C}$ and taken up in Hepes-buffer ( $1 \mathrm{mM}$ Hepes, $150 \mathrm{mM} \mathrm{NaCl}, \mathrm{pH} 7.4$, depleted from 3d-metal ions by CHELEX treatment). To identify the zincosomecontaining fraction, aliquots were labeled with TSQ and analyzed by fluorescence microscopy.

Nuclei. $5 \times 10^{7}$ cells were taken up in lysis buffer ( $10 \mathrm{mM}$ Tris/ $\mathrm{HCl}, \mathrm{pH} 7.4,15 \mathrm{mM} \mathrm{NaCl}, 60 \mathrm{mM} \mathrm{KCl}, 0.15 \mathrm{mM}$ Spermine, $0.5 \mathrm{mM}$ Spermidine, 0.5\% Nonidet P-40, 1 mM EDTA, $0.1 \mathrm{mM}$ EGTA). Cells were kept on ice for $5 \mathrm{~min}$, and nuclei were obtained by centrifugation at $600 \mathrm{~g}$ for $5 \mathrm{~min}$ at $4{ }^{\circ} \mathrm{C}$.

Cytosol. $2 \times 10^{7}$ cells were lysed in $3 \mathrm{ml}$ icecold Hepes-buffer by sonification, followed by removal of debris by centrifugation at $10.000 \mathrm{~g}$ for $10 \mathrm{~min}$ at $4{ }^{\circ} \mathrm{C}$. Small fragments were removed by filtration through a $0.22 \mu \mathrm{m}$ filter (Millipore, Cork, Ireland).

Data collection. The Zn K-edge X-ray absorption spectra on isolated subcellular fractions were recorded at the beamline D2 of the EMBL Outstation Hamburg at DESY, Germany with a Canberra 13-element Ge solid-state fluorescence detector. A Si(111) doublecrystal monochromator scanned X-ray energies around Zn K-edge. Harmonic rejection was achieved with a focusing mirror (cut-off energy at $20.5 \mathrm{keV}$ ) and a monochromator detuned to $70 \%$ of its peak intensity. The sample cells were mounted in a two-stage Displex cryostat and kept at about $20 \mathrm{~K}$. Data reduction was performed with KEMP [17] assuming a threshold energy $E_{0, \mathrm{Zn}}=9662 \mathrm{eV}$. Sample integrity during exposure to synchrotron radiation was checked by monitoring the position and shape of the absorption edge on sequential scans. No changes were detectable.

For $\mu$ XANES analysis, cells were seeded onto Kapton ${ }^{\circledR}$ foil (DuPont, Wilmington, USA) and grown under normal culture conditions for $24 \mathrm{~h}$ in the presence of $100 \mu \mathrm{M} \mathrm{ZnSO}$, to augment vesicular zinc. Afterwards samples were shock frozen in liquid nitrogen. For the $\mu \mathrm{XRF}$ - and $\mu \mathrm{XANES}$-experiments at the ESRF beamline id22, microfocusing was achieved by Pd-coated Kirkpa-
trick-Baez-mirrors with focal distance of 400 and $200 \mathrm{~mm}$, respectively. The beam size was determined during the experiment to be $2.2 \times 5.0 \mu \mathrm{m}$ at the excitation energy of $11.0 \mathrm{keV}$. The sample was mounted on a goniometer in standard $45^{\circ}$ geometry and kept under cryogenic conditions by an Oxford cryosystems cryostream series 600. A Gresham 13-element $\mathrm{Si}(\mathrm{Li})$-detector was used both for $\mu$ XRF- and $\mu$ XANES-studies [18] and its count rates corrected for deadtime effects. $\mu$ XRF-data were fitted with PyMCA [19].

EXAFS analysis. Initial evaluation of all spectra by ABRA [20] which is based on EXCURV [21] included a systematic screening of $\sim 400$ possible models. Refinement results are scored with respect to (a) goodness-of-fit (e.g., reduced $\chi^{2}$ ), (b) Debye-Waller factor, (c) bond lengths and (d) bond valence sum. The total score is based on the weighted geometrical mean of individual scores. Thus, models failing in any scoring criterion obtain a low total score. This allows the reliable determination of sulfur ligation in Zn-binding proteins [20]. Due to the very similar scattering properties, light ligands like histidine and oxygen are much more difficult to differentiate. Because of this several models might achieve very similar scores. To overcome this limitation ABRA employs a metaanalysis for these models [20]. With this approach the different cellular fractions, as well as whole cells, were analyzed and all results were compared to interactive refinements done with EXCURV. For the vesicular fraction ABRA yielded an average coordination by $0.9 \pm 0.2$ sulfur and $3.4 \pm 0.2$ light ligands. EXCURV analysis with semi-integer coordination numbers resulted in 1.0 sulfur, 2.5 histidines and 1.0 oxygen. Lower sulfur or light ligands coordination numbers result in unrealistic Debye-Waller factors far below $2 \sigma^{2}=0.004 \AA^{2}$, while higher coordination numbers would give bond valence sum values associated with an incorrect Zn-oxidation state of (III).

\section{Results and discussion}

Staining of intracellular zinc in the murine macrophage cell line RAW264.7 with the probe TSQ shows a typical distribution, with zinc-dependent fluorescence in the cytoplasm, but not the nucleus. Upon incubation with $100 \mu \mathrm{M}$ extracellular zinc for $24 \mathrm{~h}$, bright spots of fluorescence indicate the accumulation of excess zinc in zincosomes of some cells (Fig. 1). Such vesicles were isolated from unstained cells using nitrogen cavitation, after which the isolated vesicles are still stainable by TSQ, proving the presence of intact zincosomes (data not shown). Bulk Zn-edge XAS on isolated vesicles enriched in zincosomes (called 'vesicular fraction') is only sensitive to the element of interest, in this case zinc. Thus the potential presence of other, zinc-free vesicles does not influence the measurement. The presence of a beat-node at about $10 \AA^{-1}$ in the EXAFS (Fig. 2A) indicates the presence of two ligand types with different $\mathrm{Zn}$-ligand distances. This is substantiated by the corresponding Fourier-transform (Fig. 2B), highlighting one contribution at about $2 \AA$ and one at about $2.3 \AA$. In the ABRA-refinement they were identified as $0.9 \pm 0.2$ sulfur and $3.4 \pm 0.2$ light ligands (histidine or oxygen from Glu/Asp/OH/water), which is in line with the best model based on semi-integer coordination numbers indicating 1.0 sulfur (presumably cysteine) at a distance of $2.278 \AA$, 2.5 histidine and 1.0 oxygen at $1.968 \AA$.

The identification of non-water ligands shows that zinc is present in a complexed form. The average binding motif differs considerably from structural sites like zinc fingers $\left(\mathrm{Cys}_{2} \mathrm{His}_{2}, \mathrm{Cys}_{3} \mathrm{His}, \mathrm{Cys}_{4}\right)[1]$, and zinc in metallothionein $\left(\mathrm{Cys}_{4}\right)$ [11]. In contrast, in transport molecules like the bacterial YiiP, zinc is coordinated by histidine and aspartate, but no cysteine [22]. The identified ligands in zincosomes resemble in their stoichiometry regulatory (e.g., the bacterial uptake regulator Zur $[3,23]$ ) or catalytic zinc sites (e.g., farnesyltransferase [24]). Such motifs are known for their lower metal binding affinity, 

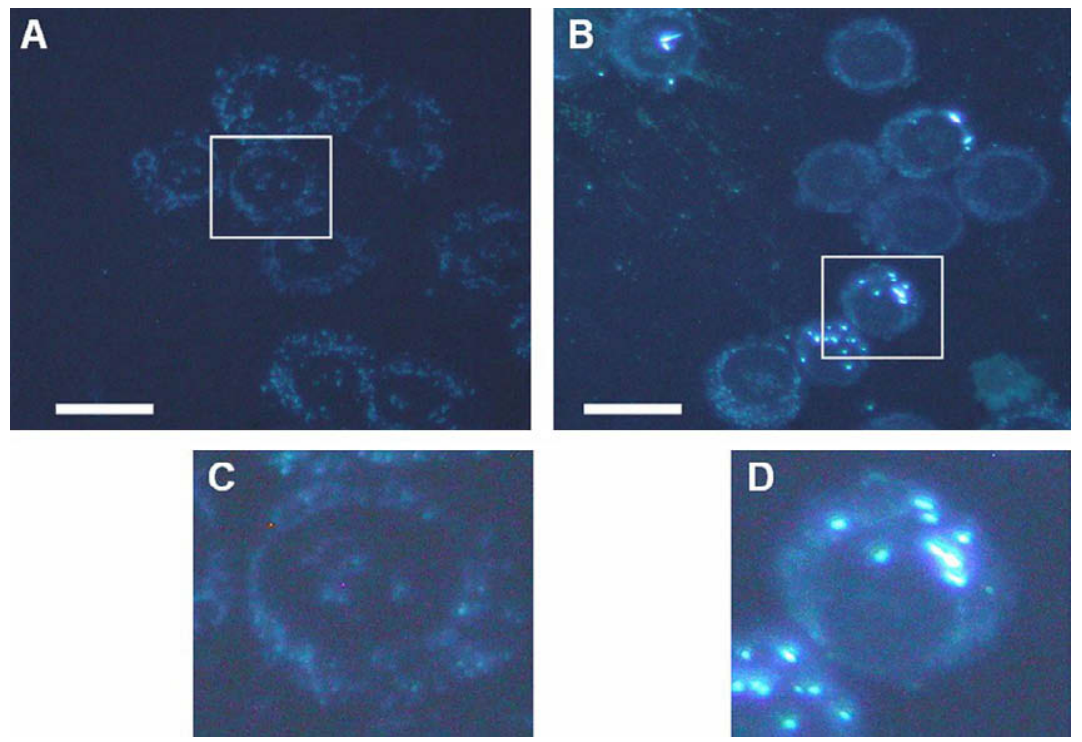

Fig. 1. Fluorescence microscopy of RAW264.7 cells stained with TSQ. Cells were grown on glass coverslips in normal culture medium (A and close-up C) or in the presence of $100 \mu \mathrm{MnSO}_{4}$ (B and close-up D). White scale bar corresponds to $10 \mu \mathrm{m}$. Vesicles enriched in $\mathrm{Zn}$ are present in several cells in B and D.
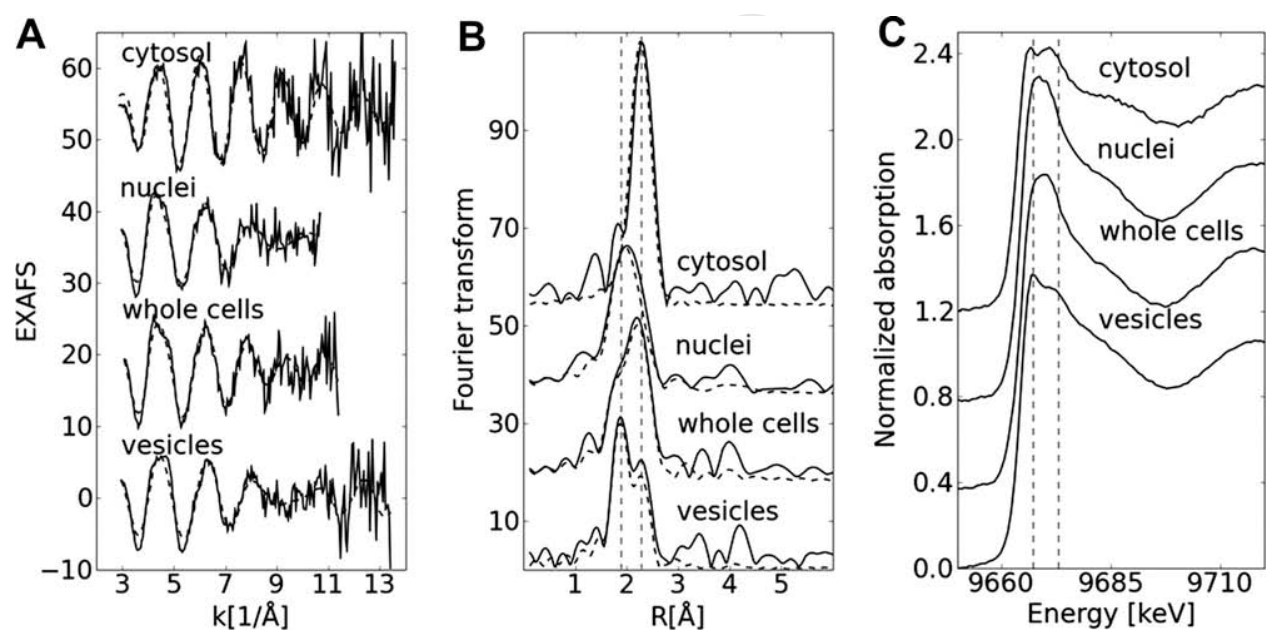

Fig. 2. (A) Extracted EXAFS spectra. The distinctive features of the vesicular /fraction (position of minima and maxima, shape of maximum at $k=5$ ) are not matched by any other spectrum. The dashed lines correspond to the structural models given in Table S1. (B) Corresponding Fourier-transformation. The peak at about $2 \AA$ originates from His/ oxygen ligands, whereas the peak at about $2.3 \AA$ is assigned to sulfur ligands. (C) XANES-spectra show distinct differences for the four fractions. Two vertical lines mark the position of peaks in the white-line of the XANES spectrum of the vesicular fraction. These features are absent in all other spectra. Vertical offset by 0.4 per spectrum.

compared to structural sites. This is in line with reversible binding and a potential release of zinc from this pool.

Moreover, this binding motif diverges from $\mathrm{Zn}$-binding patterns determined for whole cells or other cell compartments, such as nuclei and cytosol. All of them differ considerably from the spectrum of the vesicular fraction, either in the intensity of edge features, the position of the first minimum (Fig. 2C) or the position of maxima in the Fourier-transformed EXAFS (Fig. 2B). The height of the rising edge in normalized XANESspectra, named white-line, is increased for more homogeneous ligand spheres of light ligands such as oxygen and nitrogen [25]. Here, it is used together with the edge shape as a finger print, indicating vastly different average binding motifs: Vesicles exhibit a white-line intensity of 0.37 above the normalized edge jump. The peak is higher for whole cells and nuclei, 0.44 and 0.49 , respectively. For cytosol it is roughly $60-100 \%$ smaller (0.23). The spectrum of the vesicular fraction shows two distinct peaks in the white-line (indicated by two vertical lines in
Fig. 2C). Only the spectrum of cytosolic zinc exhibits features with some similarity. This is as well the case for the position of the first minimum in the XANES, which depends on the bond length of the ligands and their types [26] (9698.6 eV for the vesicular fraction and $9698.9 \mathrm{eV}$ for the cytosol spectrum). The other spectra show a significant shift to lower energies (whole cells: $9696.9 \mathrm{eV}$, nuclei: $9696.8 \mathrm{eV}$ ) indicating vastly different average binding motifs.

This differentiation is in line with the EXAFS and the corresponding Fourier-transforms shown in Fig. 2A and B, respectively. For whole cells and nuclei the spectra are dominated by the contribution at about $2 \AA$, originating from backscattering of light ligands, whereas a peak at $2.3 \AA$ ascribed to backscattering from sulfur ligands is present in both the vesicular and the cytosolic fraction. In the EXAFS analysis this observation is supported by the identification of such ligands in these two samples only (Table S1). Under the present conditions cytosolic $\mathrm{Zn}$ is bound on average as $\mathrm{Cys}_{3} \mathrm{His}_{1}$. This can be attributed to a large fraction of structural $\mathrm{Zn}$ sites or 
metallothionein-bound $\mathrm{Zn}$, where high expression of the latter protein could be caused by the $100 \mu \mathrm{M} Z \mathrm{Zn}$ concentration in the medium.

To analyze zincosomes in intact cells, RAW264.7 cells were grown onto polyimide foil in the absence of staining or fixation. Microbeam X-ray fluorescence [18] mapped the elemental distribution in several cells. The distribution of zinc is shown in Fig. 3A. In one of these cells a small region $(\sim 6 \times 4 \mu \mathrm{m})$ with a 10 -fold increase in zinc fluorescence was identified. In a similar way, the Ca concentration increases as shown in Fig. 3B whereas the amounts of other essential 3d-metals, such as Fe and $\mathrm{Cu}$ remain constant. The elemental distribution in this spot is compared with another zincosome-free cell (Fig. 3C and D). Major differences originate from the above-mentioned increase in $\mathrm{Ca}$ and $\mathrm{Zn}$ fluorescence by a factor of 10 . All other contributions originating from elements between sulfur and zinc remain roughly constant. The striking similarities in $\mathrm{Ca}$ and $\mathrm{Zn}$ concentration prompt speculations on the involvement of $\mathrm{Ca}$ in $\mathrm{Zn}$ complexation, but no spectroscopic evidence for the presence of $\mathrm{Ca}$ in the direct proximity of $\mathrm{Zn}$ was found. The correlation of $\mathrm{Ca}$ and $\mathrm{Zn}$ as seen in Fig. 3B could be caused either by a co-localization of $\mathrm{Ca}$ - and $\mathrm{Zn}$-storage vesicles or by an incorporation/storage of Ca directly in zincosomes (e.g., in order to stabilize them or because of a lack of element specificity of the transport mechanism).

Although the resolution is moderate compared to fluorescence microscopy, the accumulation of zinc can be visualized by $\mu \mathrm{XRF}$. So far, $\mu$ XAFS has already been used to analyze e.g., changes in the intracellular zinc distribution during monocytic differentiation of HL-60 cells [27], and the zinc distribution in hippocampal mossy fibers [28]. With increased spatial resolution, $\mu$ XANES could, in addition to mapping the distribution of zinc and comparing it to other elements, supply information about the ligand environment in intact cells and be a valuable tool for the analysis of zinc speciation in different cellular compartments. Even at the present resolution, analysis of the vesicular zinc speciation by $\mu$ XANES allows to exclude two potential artifacts.

Firstly, it had been suggested that zincosomes may not be vesicles at all, but may represent cytosolic protein complexes [29]. The most likely candidate for such a protein would be metallothionein, which forms oligo- and polymers linked by intermolecular disulfides [30,31]. These aggregates can still bind large amounts of zinc that is accessible to dyes, and polymerized metallothionein could be mistaken for vesicular structures. However, elemental distributions fit with a zinc-coordination by mainly light ligands, especially histidine, and significantly deviate from a binding solely by thiol-ligands as in metallothionein. In addition, the $\mathrm{Zn}$-edge $\mu$ XANES collected on the position of the zinc accumulation $(x, y=[80,42 \mu \mathrm{m}])$ in Fig. $3 \mathrm{~A}$ matches the XANES spectrum obtained from the isolated vesicular fraction (Fig. 4) but differs significantly from the cytosolic fraction in Fig. 2B. XANES patterns serve as a fingerprint for the average zinc environment, and the match between the $\mu$ XANES and the XANES spectrum obtained from the isolated vesicular fraction confirms the vesicular nature of the zinc accumulation observed in the elemental map in Fig. 3A.
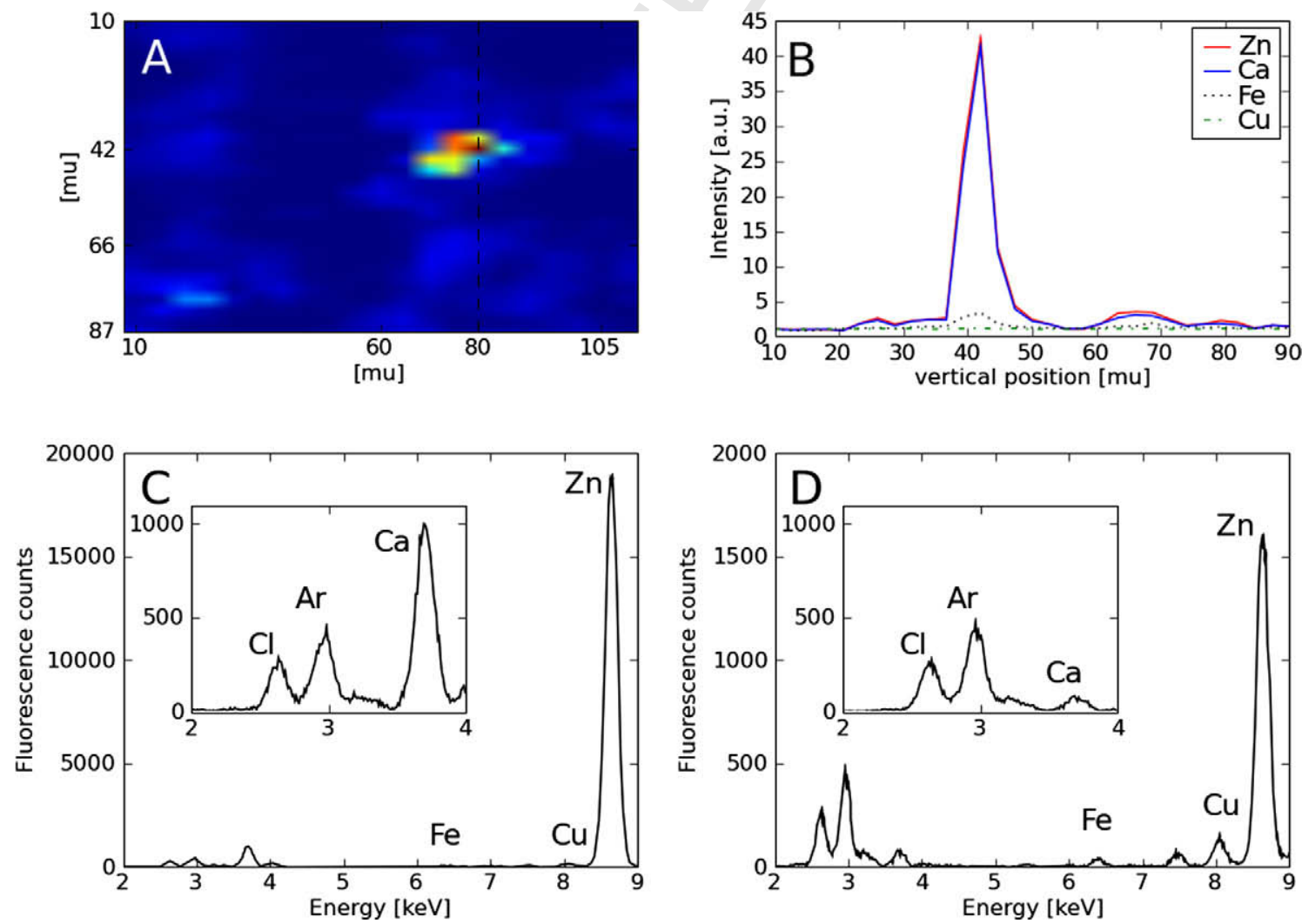

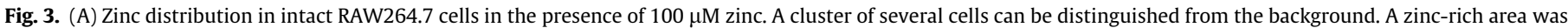

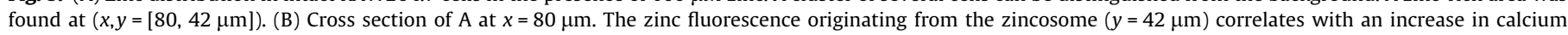

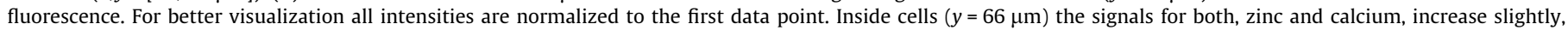

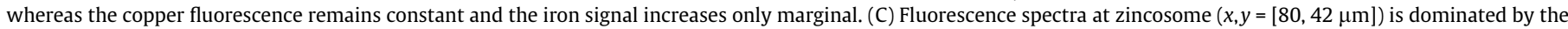

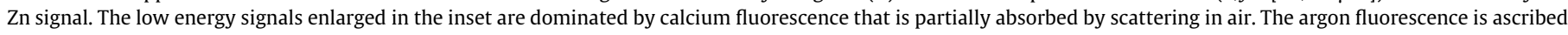

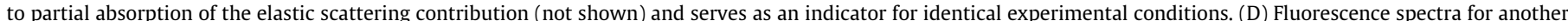

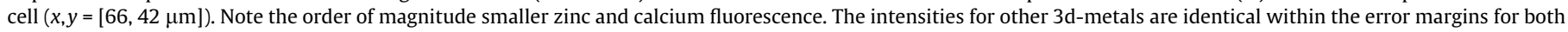
spots. 


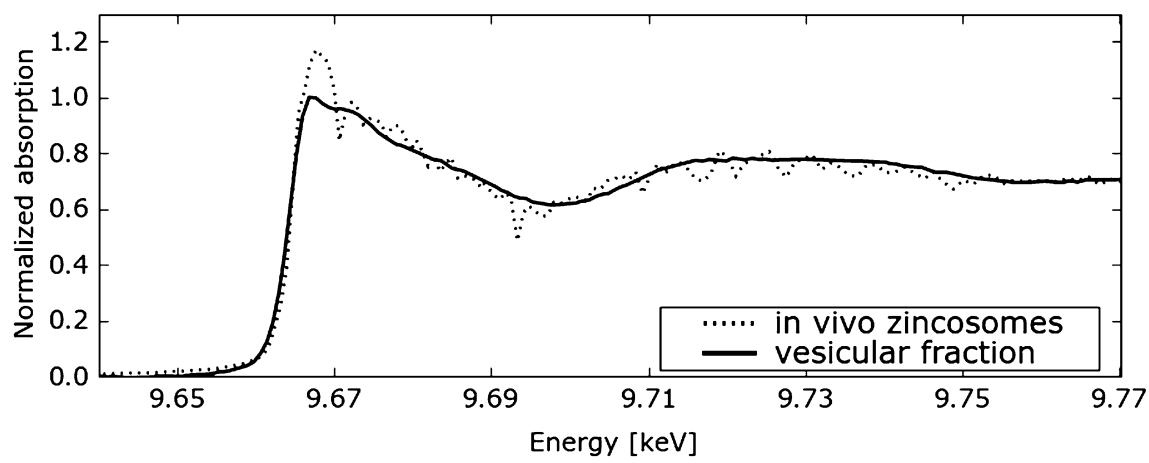

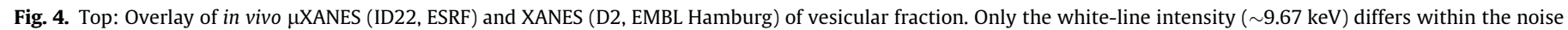
level, all other features are identical, e.g., position of the two minima as well as the elongated maximum at $9.73 \mathrm{keV}$.

Secondly, probes like Zinquin have been implied to partition inside membranes and act as an ionophore due to the relatively lipophilic nature of the dye [32]. Accordingly, zincosomes might result from an intracellular sequestration of zinc by the fluorescent probes. However, our analysis of intact cells or cellular compartments is not prone to potential artifacts resulting from dyes. Because the appearance of vesicular zinc accumulation is found in the absence of dyes (Fig. 3A), zincosomes are not an artefact due to the probe acting as an ionophore.

Control of zinc homeostasis is coupled to the cellular redox status when zinc is bound to thiols, for example in the tetraedric coordination sphere in metallothionein. Here, oxidation of the zincbinding thiol groups leads to zinc release [11]. Our results indicate the presence of a second, redox independent system, because zincosomal zinc is bound to only one sulfur per metal ion. This correlates well with previous observations whereby release of thiol bound zinc by oxidation leads to zinc accumulation in vesicles [33].

Thus, Zn-homeostasis in mammalian cells is controlled by two largely independent Zn-storage and -activation mechanisms, in which zincosomes store zinc in a complexed form, mainly bound to light, redox-inactive ligands.

\section{Acknowledgments}

We gratefully acknowledge both ESRF and EMBL Hamburg for beam time and support as well as funding by BIOXHIT project by the European Commission under FP6 contract LSHG-CT-2003503420 .

\section{Appendix A. Supplementary data}

Supplementary data associated with this article can be found, in the online version, at doi:10.1016/j.bbrc.2009.01.074.

\section{References}

[1] D.S. Auld, Zinc coordination sphere in biochemical zinc sites, Biometals 14 (2001) 271-313.

[2] J.H. Laity, G.K. Andrews, Understanding the mechanisms of zinc-sensing by metal-response element binding transcription factor-1 (MTF-1), Arch. Biochem. Biophys. 463 (2007) 201-210.

[3] D. Lucarelli, S. Russo, E. Garman, A. Milano, W. Meyer-Klaucke, E. Pohl, Crystal structure and function of the zinc uptake regulator FurB from Mycobacterium tuberculosis, J. Biol. Chem. 282 (2007) 9914-9922.

[4] L. Rink, H. Haase, Zinc homeostasis and immunity, Trends Immunol. 28 (2007) $1-4$.

[5] H. Haase, L. Rink, Functional significance of zinc-related signaling pathways in immune cells, Annu. Rev. Nutr. 29 (2009), doi:10.1146/annurev-nutr-080508 141119.

[6] L.A. Finney, T.V. O'Halloran, Transition metal speciation in the cell: insights from the chemistry of metal ion receptors, Science 300 (2003) 931-936.
[7] R.J. Cousins, J.P. Liuzzi, L.A. Lichten, Mammalian zinc transport, trafficking, and signals, J. Biol. Chem. 281 (2006) 24085-24089.

[8] D.J. Eide, Zinc transporters and the cellular trafficking of zinc, Biochim. Biophys. Acta 1763 (2006) 711-722.

[9] A. Krezel, W. Maret, Dual nanomolar and picomolar Zn(II) binding properties of metallothionein, J. Am. Chem. Soc. 129 (2007) 10911-10921.

[10] E.A. Peroza, A.A. Kaabi, W. Meyer-Klaucke, G. Wellenreuther, E. Freisinger, The two distinctive metal ion binding domains of the wheat metallothionein $\mathrm{E}(\mathrm{c})-$ 1, J. Inorg. Biochem. (2008).

[11] W. Maret, Zinc coordination environments in proteins as redox sensors and signal transducers, Antioxid. Redox Signal. 8 (2006) 1419-1441.

[12] P.D. Zalewski, I.J. Forbes, W.H. Betts, Correlation of apoptosis with change in intracellular labile $\mathrm{Zn}$ (II) using zinquin [(2-methyl-8-p-toluenesulphonamido6-quinolyloxy)acetic acid], a new specific fluorescent probe for $\mathrm{Zn}(\mathrm{II})$, Biochem. J. 296 (1993) 403-408.

[13] C.J. Frederickson, S.W. Suh, D. Silva, C.J. Frederickson, R.B. Thompson, Importance of zinc in the central nervous system: the zinc-containing neuron, J. Nutr. 130 (2000) 1471S-1483S.

[14] H. Haase, W. Maret, Protein tyrosine phosphatases as targets of the combined insulinomimetic effects of zinc and oxidants, Biometals 18 (2005) 333-338.

[15] P. Coyle, P.D. Zalewski, J.C. Philcox, I.J. Forbes, A.D. Ward, S.F. Lincoln, I. Mahadevan, A.M. Rofe, Measurement of zinc in hepatocytes by using a fluorescent probe, zinquin: relationship to metallothionein and intracellular zinc, Biochem. J. 303 (1994) 781-786.

[16] A.K. Schroder, O.M. von der, D. Fleischer, L. Rink, P. Uciechowski, Differential synthesis of two interleukin-1 receptor antagonist variants and interleukin-8 by peripheral blood neutrophils, Cytokine 32 (2005) 246-253.

[17] M. Korbas, D.F. Marsa, W. Meyer-Klaucke, A program script for automated biological x-ray absorption spectroscopy data reduction, Rev. Sci. Instrum. 77 (2006) 063105

[18] I. Letard, R. Tucoulou, P. Bleuet, G. Martinez-Criado, A. Somogyi, L. Vincze, J. Morse, J. Susini, Multielement $\mathrm{Si}(\mathrm{Li})$ detector for the hard X-ray microprobe at ID22 (ESRF), Rev. Sci. Instrum. 77 (2006) 063705.

[19] V.A. Sole, E. Papillon, M. Cotte, P. Walter, J. Susini, A multiplatform code for the analysis of energy-dispersive X-ray fluorescence spectra, Spectrochim. Acta B Atom Spectrosc. 62 (2007) 63-68.

[20] G. Wellenreuther, W. Meyer-Klaucke, Towards a black-box for biological EXAFS data analysis-I. Identification of zinc finger proteins, AIP Conf. Proc. 882 (2007) 322-324.

[21] S. Tomic, B.G. Searle, A. Wander, N.M. Harrison, A.J. Dent, J.F.W. Mosselmans, J.E. Inglesfield, New tools for the analysis of EXAFS: the DL EXCURV Package, CCLRC Tech. Rep. (2005) 1-10.

[22] M. Lu, D. Fu, Structure of the zinc transporter YiiP, Science 317 (2007) 17461748.

[23] C.E. Outten, D.A. Tobin, J.E. Penner-Hahn, T.V. O'Halloran, Characterization of the metal receptor sites in Escherichia coli Zur, an ultrasensitive zinc(II) metalloregulatory protein. Biochemistry 40 (2001) 10417-10423.

[24] D.A. Tobin, J.S. Pickett, H.L. Hartman, C.A. Fierke, J.E. Penner-Hahn, Structural characterization of the zinc site in protein farnesyltransferase, J. Am. Chem. Soc. 125 (2003) 9962-9969.

[25] A. Mijovilovich, W. Meyer-Klaucke, Simulating the XANES of metalloenzymes-a case study, J. Synchrotron. Radiat. 10 (2003) 64-68.

[26] P. D’Angelo, A. Lapi, V. Migliorati, A. Arcovito, M. Benfatto, O.M. Roscioni, W. Meyer-Klaucke, S. Della-Longa, X-ray absorption spectroscopy of hemes and hemeproteins in solution: multiple scattering analysis, Inorg. Chem. 47 (2008) 9905-9918.

[27] D. Glesne, S. Vogt, J. Maser, D. Legnini, E. Huberman, Regulatory properties and cellular redistribution of zinc during macrophage differentiation of human leukemia cells, J. Struct. Biol. 155 (2006) 2-11.

[28] D.H. Linkous, J.M. Flinn, J.Y. Koh, A. Lanzirotti, P.M. Bertsch, B.F. Jones, L.J. Giblin, C.J. Frederickson, Evidence that the ZNT3 protein controls the total amount of elemental zinc in synaptic vesicles, J. Histochem. Cytochem. 56 (2008) 3-6. 
[29] R.A. Colvin, M. Laskowski, C.P. Fontaine, Zinquin identifies subcellular compartmentalization of zinc in cortical neurons. Relation to the trafficking of zinc and the mitochondrial compartment, Brain Res. 1085 (2006) $1-10$.

[30] H. Haase, W. Maret, A differential assay for the reduced and oxidized states of metallothionein and thionein, Anal. Biochem. 333 (2004) $19-26$
[31] H. Haase, W. Maret, Partial oxidation and oxidative polymerization of metallothionein, Electrophoresis 29 (2008) 4169-4176.

[32] V. Snitsarev, T. Budde, T.P. Stricker, J.M. Cox, D.J. Krupa, L. Geng, A.R. Kay, Fluorescent detection of $\mathrm{Zn}(2+)$-rich vesicles with Zinquin: mechanism of action in lipid environments, Biophys. J. 80 (2001) 1538-1546.

[33] H. Haase, D. Beyersmann, Intracellular zinc distribution and transport in C6 rat glioma cells, Biochem. Biophys. Res. Commun. 296 (2002) 923-928.

Please cite this article in press as: G. Wellenreuther et al., The ligand environment of zinc stored in vesicles, Biochem. Biophys. Res. Commun. (2009), doi:10.1016/j.bbrc.2009.01.074 